awaited, authoritative and occasionally pungent review by Edman of the stepwise degradation method that bears his name. Procedures refined by the author over a period of 20 years, culminating in the automatic protein sequenator, are described in detail. Edman gives cogent reasons for preferring positive identification of amino-acid PTHs to subtractive procedures, and this opinion is reinforced by recent developments in the separation and identification of these compounds by GLC and mass spectrometry. Nevertheless, the brief treatment of the dansyl-Edman technique in Narita's otherwise comprehensive survey of $N$ and $C$-terminal methods hardly reflects its present utility and popularity. The fiuoronitropyridine reagents developed by the Padua school might have been referred to in preference to some of the "Other Chemical Methods", and the use of polyamide layers, currently one of the best methods for separating DNSamino-acids, is not mentioned.

Von Holde gives a useful summary of physical methods for the characterization of proteins, and Eveleigh and Winter write in a practical way about up-to-date methods of amino-acid analysis, with special reference to Technicon instruments. Linenberg's short chapter on amino-acid analysis by gas chromatography has inevitably been rather left behind by this fast-developing field. Kasper contributes a chapter on methods for peptide bond fission and the separation of mixtures of peptides. Cyanogen bromide, dilute and concentrated acids, and the more specific enzymes are adequately dealt with, but there is no mention of subtilisin, thermolysin, elastase nor "Pronase". Peptide mapping is not described despite a cross-reference on p. 207 (indexed as p. 206), and diagonal electrophoresis gets only a brief mention.

The theme of the chapter by Scoffone and Fontana is the specific reactivity of particular amino-acid residues which serves for their determination and also their detection on paper and other media. Quantitative methods for total protein, tryptophan, cystine and cysteine are given in some detail. The description of disulphide bond reduction and alkylation (p. 202) is duplicated by Kasper (p. 152, where ref. (318) should be (218)). This typifies both a certain amount of repetition that could have been reduced by careful cross-referencing, and too great a prevalence of trivial errors. Goldstone and Needleman discuss the reconstruction of the primary sequence from peptide fragments. The application of computers is described, but the pairing of half-cystine residues is not dealt with. Gish contributes a chapter on peptide synthesis which, as far as I can judge, gives a balanced account of the subject. A useful addi- tion would have been an account of the synthesis of side-chain-substituted derivatives of amino-acids, particularly lysine and cysteine, which are often encountered in sequence work but may not be easily available. There is no contribution dealing with the use of the mass spectrometer for the determination of sequences.

Although many laboratories will want this book primarily for the chapter by Edman, it does as a whole cover a large part of present-day sequence methodology. But only those who take the trouble to thumb through it and familiarize themselves with the contents will be able to make full use of it as a work of reference, and newcomers to the field would be unwise to rely on it exclusively.

$$
\text { J. C. Fletcher }
$$

\section{Oxide Compendium}

Landölt-Bornstein. Numerical Data and Functional Relationships in Science and Technology. New Series. Editor in Chief: K. H. Hellwege. Group III: Crystal and Solid State Physics. Vol. 4: Magnetic and Other Properties of Oxides and Related Compounds, Part b. By D. Bonnenberg et al. Editors: K. H. Hellwege and A. M. Hellwege. Pp. xvi+ 666. (Springer-Verlag: Berlin and New York, 1970.) $428 \mathrm{DM}$; \$117.70.

THis book, which completes the fourth part of Volume III of these tables, contains information about three classes of compounds-non-iron garnets, spinels, and hexagonal ferrites. It is a composite work by authors from four different countries-Germany, the United States, France and the Netherlands-and no trouble has been spared to make it comprehensive. Results are published in the form of tables and diagrams, and every conceivable measurement of physical properties seems to have been included together with extensive lists of references.

The compounds are characterized by simple atomic arrangements in which extensive substitution is possible, so that an infinite variety of compositions exists. Each section is preceded by a concise but clear introduction in both English and German, and it is obvious that the authors are more concerned with the fundamental importance of the results than with their practical application. One would have thought that results as extensive as these would have been acquired only if there were some important applications, and this is hinted at, for example, in a diagram on p. 278, which shows an area of a ternary oxide system that is important in microwave research. More indications of this sort would have been welcome.

The book is well indexed in terms of the chemical formulae of the compounds included. It would also have been helpful if an index of physical properties had also been produced; one can imagine a research project that might require a material with a particular property, and it would require a great deal of searching to find the possibilities from the mass of data included.

The book is impeccably produced in accordance with the well known LandöltBornstein tradition. The diagrams are clear and informative and the legends, although only in the language of the originators, are sufficiently simple to be readily understood with minimal linguistic ability. The tables are well set out and uncluttered. Altogether, the book should form a standard work of reference for those whose interests lie in the compounds included.

H. LIPSON

\section{Mössbauer Collection}

Proceedings of the Conference on the Application of the Mössbauer Effect, Tihany, Hungary, 17th/21st June, 1969. Edited by I. Dezsi. Pp. 808. (Akademiai Kiado: Budapest, 1971.) $£ 11$.

THIs book is a collection of ninety-two specialist papers presented at the 1969 Tihany Conference on the Mössbauer Effect. Most of the papers are from East European research groups, so that they give a somewhat one-sided view of the current state of research. All of them have been translated into English, the standard of which is usually good. The chief value of the volume is that it brings together, under one cover, reports of the work of research groups that are not always easily obtained in English.

A comprehensive picture of the wide range of applications of the Mössbauer effect is given by the range of topics covered, divided into the following sections: General Problems, Relaxation Effects, Goldanskii-Karyagin Effect, Surface Phenomena, Alloys, Oxides, Frozen Solutions, Biology, Chemistry and Miscellany. Although there are brief review papers at the start of most of the sections, there is unfortunately no general introduction to the Mössbauer effect nor reference to the many excellent introductory texts now available for the non-specialist The review papers in biology by G. Long and chemistry by C. E. Johnson are particularly clear and well presented.

Among the individual papers, those by A. H. Muir and M. Blander (extra terrestrial materials), M. A. Andreeva and R. N. Kuzmin (narrowing of Mössbauer lines) and V. D. Gorobchenko et al. (stabilization of hyperfine structure in corundum by a small external magnetic field) are particularly noteworthy. It is, however, a pity that in general many of the spectra on which detailed discussions are made are of such a relatively poor quality. Often a modest increase in running time would have yielded many more convincing data. F. W. D. Woodhams 